\title{
Fifth meeting of the International Society for STD Research
}

\author{
Report on the fifth meeting of the International Society for STD Research held on 1-3 August 1983 in Seattle, \\ Washington, USA.
}

\section{Clinical update course}

The fifth meeting of the International Society for STD Research was held at the Seattle Sheraton Hotel on 1-3 August 1983. It was preceded by a clinical update course on Sexually Transmitted Diseases (STDs) held on . 31 July 1983 at the same hotel. About 300 participants took part in a lively meeting in which the current state of knowledge of several sexually transmitted diseases and syndromes were reviewed by experts in their particular fields. The standards of the papers and their presentation was very high and many lively discussions had to be curtailed by the chairman because of lack of time.

Dr King Holmes of Seattle reviewed the whole field of STDs, and suggested that we might now be seeing the emergence of a third generation of STDs, of which the acquired immune deficiency syndrome (AIDS) was the first and most striking example. He also drew attention to the recent clarification of several STD syndromes such as genital ulcer disease, lower genital tract infection in women, pelvic inflammatory disease, ectopic pregnancy and infertility, fetal and neonatal infections, and genital neoplastic disease and its relation to certain viral infections. The review was very detailed but only included conditions which were increasing in incidence or remaining very prevalent. For example, no mention was made of the virtual disappearance

Address for reprints: Dr R D Catterall, 61 Durrels House, Warwick Gardens, London W14 8QB

Accepted for publication 12 September 1983 of late syphilis which is one of the major advances in the health of mankind.

Dr Frank Judson of Denver reviewed the diagnosis of gonorrhoea and described some of the advantages of combined antibiotic treatment. To ensure that chlamydial infections did not escape treatment he recommended using either amoxycillin, ampicillin, or aqueous procaine penicillin with probenecid in a single dose, and either tetracycline or doxycycline for seven days. There was considerable discussion about the desirability and efficacy of using several different antibiotics, and some speakers thought it was most undesirable.

Dr Julius Schachter of San Francisco pointed out that the only way of making the diagnosis of chlamydial infection was by culture, and in a very detailed and thorough review of the subject stressed the fact that this agent was responsible for $35-50 \%$ of all cases diagnosed as non-gonococcal urethritis. He drew attention to the granular proctitis caused by chlamydiae which could be diagnosed from carefully taken rectal swabs. The role of chlamydiae in epididymitis and male infertility had not yet been investigated, and the question had been recently raised as to whether chlamydiae were often the cause of sterile urinary tract infections.

In a rather confused and confusing review of syphilis, Dr Peter Perine of Atlanta drew attention to the fact that it was still the third most prevalent infectious disease in the United States, and was increasing in incidence in homosexual men in many areas of the world. Dr Lawrence Corey of Seattle gave an exhaustive review of genital herpes simplex infections, and stressed the importance of recognising first $\stackrel{\vec{\circ}}{\circ}$ episodes. He wondered whether the $\vec{\omega}$ increased prevalence of genital herpes ? could be due to a decrease in oral $\stackrel{?}{=}$ herpes simplex, and gave practical 8 details of the management of pregnant i women with the disease. Professor $\mathrm{E}_{\vec{N}}$ Stolz of Rotterdam showed a series of $c$ transparencies to illustrate the differ-음 ential diagnosis of genital ulcerative disease. Dr David Oriel of London $\frac{D}{O}$ then gave a compact account of urethritis in men, and advocated the use of $\vec{\theta}$ tetracycline with penicillin in the treat- $\stackrel{\infty}{\perp}$ ment of gonorrhoea. The increasing complexity of vaginal discharges and the large number of micro-organisms associated with them was described by Dr Michael Rein of Charlottesville.

Dr Lars Weström of Lund continued $\cong$ to unravel the details of his 20-year $\overrightarrow{\vec{O}}$ study of pelvic inflammatory disease 3 (PID) of which $75 \%$ of cases are probably due to STD. Risk factors are little understood but include exposure $\stackrel{\text { ? }}{?}$ to STD, the use of intrauterine contraceptive devices, youth, economic 3 . status, and previous episodes of PID. $\delta$ Oral contraceptive pills may protect $₹$ women from PID. Cultures from the cervix give no reliable information $\rightarrow$ about the aetiology of tubal infection, 음 whose sequelae are very serious-one in five affected women become infer-or tile, one in six develop chronic ab- $N$ dominal pain, and one in 16 have an N ectopic pregnancy. Doctor Weström pointed out that treatment was dis- 0 appointing, and the infertility rate is the same whatever the antibiotic used. By the time the patient reaches the doctor it is usually too late to prevent $\overline{0}$ sterility in a high proportion of cases, $\stackrel{\mathbb{D}}{\stackrel{D}{ }}$ which shows that early diagnosis and $\frac{P}{\mathbb{D}}$ treatment of genital infections is $\frac{\circ}{\sigma}$ essential. 
Dr Walter Stamm of Seattle presented a very detailed study of enteric infections in homosexual men. The most important factors in transmission were multiple partners, sexual practices involving faeco-oral contact, asymptomatic infections, limited availability of diagnostic tests, blind non-specific treatment, and failure of contact tracing. He described three distinct clinical syndromes-proctitis, proctocolitis, and enteritis-which could be distinguished by proctoscopy and sigmoidoscopy. Proctitis was caused by Neisseria gonorrhoeae, herpes, chlamydiae, or Treponema pallidum; proctocolitis by Entamoeba histolytica, Shigella spp, Clostridium difficile, lymphogranuloma venereum or Campylobacter spp; and enteritis was caused primarily by Giardia lamblia. In clinics attended by many homosexual men a sigmoidoscope, and staff trained to use it, are essential as the procedure enables the conditions to be classified into definite syndromes.

Many people were waiting to hear the most up to date information about AIDS, and when Dr Hunter Hansfield of Seattle spoke on the subject the hall was filled to capacity. AIDS was first recognised in 1981 in the United States, and by July 1983 there had been 1902 reported cases and 732 deaths $(38 \cdot 5 \%)$. Every six months since 1981 the number of cases has doubled, and 755 cases were reported to the Center for Disease Control (CDC) in Atlanta between January and June 1983. The syndrome was most common in people aged 20 to 49 , and $46 \%$ of patients are between 30 and 39 . The highest incidence was in New York, San Francisco, Newark, and Los Angeles. The patients at greatest risk included homosexually active men, intravenous drug users, Haitians, haemophiliacs, and recipients of other blood products; and patients presented most commonly with pulmonary disease, tumours of Kaposi's sarcoma, or both, and other opportunistic infections. Kaposi's sarcoma was found in over $50 \%$ of homosexual men and only $4 \%$ of drug addicts with AIDS. Diagnosis is confirmed by skin biopsy tests and by abnormalities of $\mathrm{T}$ lymphocyte subsets and cutaneous anergy.

There are two main hypotheses about the cause of AIDS. The first postulates that it is due to a specific agent or micro-organism which is frequently sexually transmitted, and the second that the condition in homosexual men is due to multiple antigenic exposures to repeated infections which lead to the suppression of cellular immunity. There is no evidence that medical staff, either those in direct contact with patients or hospital workers associated with the hotel aspects of hospital services, have been infected. Worries have been expressed about the danger to those receiving hepatitis B vaccine, but it was pointed out that no case had been reported in over half a million people vaccinated, although ten cases have been reported after blood transfusions. Spread of the syndrome by personal, non-sexual contact was unlikely, and homosexual men should be advised to reduce their sexual activity and stick to one sexual partner, at least until the cause of AIDS and its management have been fully worked out. The paper provoked detailed discussion, and the meeting ended late because of the barrage of questions and comments which followed.

The day was most successful, with papers of a high standard presented by experienced speakers giving up to date information, some of which was available for the first time. Those who attended the update will be pleased they did so, and those who were unable to be present missed a model of postgraduate teaching and exchange of information. The day ended with a reception in the Seattle Aquarium.

\section{Fifth international meeting}

The fifth international meeting of the International Society for STD Research started with a symposium on AIDS. Dr James Curran of Atlanta said that since the first recognition of AIDS there had been an unprecedented mobilisation of resources in the United States to try to establish its cause. The publicity had enhanced public awareness of STD, but there had been epidemic of hysteria about AIDS. He thought that genitourinary docto $\vec{s}$ with their wider interests and trainitg were in the best position to undertake research work into the condition, a that we may only be seeing the tip the iceberg of cases. He enumerated the risk factors, which were consistent with the model of STDs such $\vec{s}$ hepatitis B. He thought that the priorities for research were to identi $\overrightarrow{\mathrm{fy}_{\mathrm{f}}}$ the aetiological agent, study the development of the disease, and monitor epidemiological dat Anxious homosexual men should be reassured and advised to avoid sexurgi activity with men known to have the disease. They should reduce the number of sexual partners to one, ang should not give blood for transfusi in until further progress had been made in understanding AIDS.

Dr Anthony Fauci of Bethesda discussed the theories of the cause ang development of AIDS in relationsh to $T$ cell function, and Dr Kenneth Se্dㄱ of the National Institute of Healgh . pointed out that only 20 cases wefe related to the transfusion of blood and none had been reported as a result \&f needle stick accidents. Among the 120 AIDS patients admitted to hospital far none had been medical staff, and the risks to those looking after estab-. lished cases seemed to be very small. $\mathrm{He}$ reviewed at some length the possibie causes, but stated that despite a greast deal of work the cause was completely unknown.

At this stage the meeting dividert into two sections, one discussing infee. tions in homosexual men and the othef the biology of Neisseria gonorrhoegs and the pathogenesis of gonococc infections. Important papers, included one from Dr D H Ostrow from Chicage who reported on a small group $\leftrightarrow$ homosexual men who did not resporye to the Merck hepatitis B vaccine and were refractory to stimulation by othe antigens. Doctor J E Berger of Seattie discussed the aetiology of epididymitis in sexually active heterosexual an homosexual men. Escherichia coli ws 
the commonest cause in heterosexual men over 35 , and sexually transmitted agents in those under 35. Coliforms and other enteric organisms were the commonest causes in homosexual men of all ages. A plenary poster session followed, at which a large number of excellent posters were displayed and discussed.

A lunch time speech by Dr Richard M Krause, the director of the National Institute of Allergy and Infectious - Diseases, entitled "STD research as seen through the prism of history" was characterised by a broad discussion of the relevance and direction of much modern research, and some important pointers for the future. In the evening there was a crowded boat journey to an island in the bay for an excellent salmon bake and display of red indian dances.

The next day a large number of excellent and well illustrated papers were presented. Dr Stanley Falcow of Stamford discussed the development and use of new molecular biological techniques for the diagnosis and treatment of STD, and showed some pictures of plasmid induced molecular magic. Dr R Nowinski of Seattle described the use of monoclonal antibodies for the rapid diagnosis of STD and painted a vivid picture of things to come. The sections on infections with chlamydia and Haemophilus ducreyi both contained new information, and discussion of $T$ pallidum ranged from in vitro cultivation to the indications for lumbar puncture in diagnosis and as a test of cure. The afternoon poster session was as varied and impressive as on the previous day, and small groups of people remained until the early evening discussing the contents of some of the demonstrations.

At lunch time there was a meeting of the American Venereal Diseases Association and the Thomas Parran award was presented to Dr King $\mathrm{K}$ Holmes of Seattle, who gave an address on medical education in STD in the United States. In the evening a banquet was held in the grand ballroom of the Sheraton Hotel.

There was a large crowd at the Wednesday morning plenary session on sexually transmitted virus infections, at which Dr R U Levine of New York discussed the male consorts of women with warts and Dr J D Oriel of London discussed cervical epithelial abnormalities in women with vulval warts. Dr Y Bryson of Los Angeles described the natural history of herpes, and several papers on acyclovir in its treatment were read. Dr Frank Judson of Denver described some ingenious techniques to show that condoms provide an effective barrier against the spread of Chlamydia trachomatis and herpes simplex virus. Dr J Douglas of Seattle presented information on the use of prophylactic oral acyclovir for frequent recurrences of genital herpes.

The afternoon plenary session on salpingitis and infertility included excellent papers on the aetiology and diagnosis of salpingitis by $\operatorname{Dr} \underset{\varnothing}{\varnothing}$ Brihmer of Stockholm, and by Dr I Svensson of Lund on contraceptiveş? and acute salpingitis.

The final session was a discussion of treatment of STD, and after three general papers a panel discussed thetreatment recommendations of the CDC in Atlanta and the WHO. NQ definite recommendations were made but important subjects such as $\beta$-lactamase producing strains of gono= cocci, the new $\beta$-lactam antibiotics $\vec{\omega}$ and the treatment of genital ulces disease were discussed in detail.

The meeting ended at $5 \mathrm{pm}$ on Wed nesday, 3 August. There were over 700 registered delegates, the overall stand $\overrightarrow{0}$ ard of presentation of papers was high and the poster sessions contained $\underset{\mathfrak{a}}{ }$ mine of important facts and new dis $\vec{\nabla}$ coveries. The growing importance of STD in modern life, especially insexually active young people, is weldo illustrated by the increasing success + size, and quality of meetings of the International Society for STD research and the veritable cascade of informa tion and new facts which emerged during the three crowded days of the conference. Assimilation of these data will take much longer. The organisers are to be congratulated on the effici 5 ency of the planning and the detaile execution of the various parts of the conference. The next meeting will be held in London in the summer of 1985 윽

$R$ C Catterat

Vice President, IUVDT 